\title{
Feedforward/Feedback Control of Multivariable Nonlinear Processes
}

This paper concerns general MIMO nonlinear processes, whose dynamic behavior is described by a standard state-space model of arbitrary order, including measurable disturbances. The concept of relative order of an output with respect to an input, extended to include disturbance as well as manipulated inputs, is generalized in a MIMO context and it is used to obtain a characterization of the dynamic interactions among the input and the output variables. A synthesis formula is calculated for a feedforward/state feedback control law that completely eliminates the effect of the measurable disturbances on the process outputs and induces a linear behavior in the closed-loop system between the outputs and a set of reference inputs. The input/output stability and the degree of coupling in the closed-loop system are determined by appropriate choice of adjustable parameters. A MIMO linear controller with integral action completes the feedforward / feedback control structure. The developed control methodology is applied to a continuous polymerization reactor and its performance is evaluated through simulations.

\section{Introduction}

Chemical processes, multivariable in nature, exhibit as a rule a highly nonlinear and highly interacting behavior. Efficient control of such processes is necessary, especially under the recently increasing environmental, operational and energy restrictions. In fact, multivariable control has emerged as a major research area during the last 20 years. The huge existing literature (for a survey see Ray, 1983) offers a variety of methodologies, both from a state-space and from an input/ output perspective. The majority of these methodologies, however, is based on a linear approximation of the nonlinear process, which is valid only in a small neighborhood around the operating steady state. For this reason, the presence of severe nonlinearities translates into large robustness margins in the linear controller design, leading therefore to conservatism and, in some cases, to extremely poor performance. On the other hand, the inherent complexity of MIMO nonlinear systems makes it almost impossible to use empirical methods to synthesize nonlinear controllers of acceptable generality for set point tracking and disturbance rejection.

On the nonlinear systems theory front, transparent solutions for typical multivariable control problems were obtained only recently which include: invertibility (Hirschorn, 1979); decoupling via state feedback (Freund, 1975; Ha and Gilbert, 1986); noninteracting control and disturbance decoupling (Hirschorn, 1981; Isidori et al., 1981); exact state equation linearization
(Hunt et al., 1983); input/output linearization (Kravaris and Soroush, 1990). It was the differential geometric framework that allowed an elegant formulation and geometric interpretation of these problems and their solutions. Research in this area has reached such a point that the synthesis of nonlinear compensators for stabilization, set point tracking, and disturbance rejection can and must be addressed in a unified framework. At the same time, recent advances in model development from first principles and increasing computational capabilities encourage the development of such a framework in this direction.

In this work, we present a unified methodological framework for the feedforward/state feedback control of a large class of multivariable nonlinear processes, with the main emphasis on the regulatory aspect of the problem. While the main result is a generalization of a previous result for SISO systems (Daoutidis and Kravaris, 1989a), the proposed methodology naturally identifies the fundamental nature of the multivariable control problem and provides a synthesis framework to address it.

In particular, we consider general MIMO nonlinear processes with a state-space description of the form:

$$
\begin{aligned}
& \dot{x}=f(x)+\sum_{j=1}^{m} g_{j}(x) u_{j}+\sum_{k=1}^{p} w_{k}(x) d_{k} \\
& y_{i}=h_{i}(x), \quad i=1, \ldots, m
\end{aligned}
$$

where $f, g_{j}, w_{x}$ are smooth vector fields on $\mathbb{R}^{n}, h_{i}$ are smooth scalar fields on $\mathbb{R}^{n}$, and 


$$
x=\left[\begin{array}{c}
x_{1} \\
x_{2} \\
\cdot \\
\cdot \\
x_{n}
\end{array}\right] \in \mathbb{R}^{n}, d=\left[\begin{array}{c}
d_{1} \\
d_{2} \\
\cdot \\
\cdot \\
\cdot \\
d_{p}
\end{array}\right] \in \mathbb{R}^{p}, u=\left[\begin{array}{c}
u_{1} \\
u_{2} \\
\cdot \\
\cdot \\
\cdot \\
u_{m}
\end{array}\right] \in \mathbb{R}^{m}, y=\left[\begin{array}{c}
y_{1} \\
y_{2} \\
\cdot \\
\cdot \\
\cdot \\
y_{m}
\end{array}\right] \in \mathbb{R}^{m}
$$

are the vectors of the states, the disturbance inputs, the manipulated inputs and the outputs (to be controlled), respectively, with $m \leq n$. For simplicity, we assume equal number of manipulated inputs and controlled outputs.

The general servo and regulatory control problem for such processes will be addressed and solved in two steps, in analogy with the SISO case (Daoutidis and Kravaris, 1989a) and the disturbance-free MIMO case (Kravaris and Soroush, 1990). At the first step, which is the main synthesis problem, all the available process information (i.e., model structure and parameters, measurements of the states and the disturbances) will be used to synthesize a feedforward/state feedback control law which:

- Eliminates the effect of measurable disturbances on the process outputs $y_{i}$ 's.

- Induces a prespecified linear behavior between the outputs and a set of reference inputs $v_{j}$ 's.

Once this synthesis problem is solved, the second step involves using linear control theory to design a linear MIMO controller with integral action around the linear $v-y$ system. This will ensure satisfactory servo and regulatory behavior, despite the presence of unmeasured disturbances and/or model uncertainty. The overall control configuration is shown in Figure 1 and it clearly depicts the resulting two-step control methodology.

We will begin by introducing a generalization of the concept of relative order in a MIMO setting to incorporate disturbance as well as manipulated inputs. The fundamental nature of the regulatory control problem will then be discussed, using the relative order as a structural analysis tool. In the following section, the main theoretical result will be developed, i.e., a feedforward/state feedback control law that solves the posed synthesis problem. This will lead to the development of a feedforward/state feedback control structure. An alternative feedforward/output feedback control structure will be proposed for the case of unavailable state measurements. A discussion will follow, in which specific design objectives in the closed-loop system will be associated with the choice of some adjustable parameters. Finally, the developed feedforward/feedback methodology will be applied to a continuous polymerization reactor and its performance will be evaluated through simulations.

\section{Relative Order and the Fundamental Nature of the Control Problem}

An extension of the concept of relative order to include disturbance inputs as well as manipulated inputs was recently introduced in the context of SISO systems (Daoutidis and Kravaris, 1989a); it was proven to be a very useful tool in analyzing the dynamic structure of a SISO nonlinear system and developing a feedforward/feedback control methodology. A similar extension will now be introduced in the context of MIMO systems and will be used to characterize the nature of the regulatory control problem, based on a structural analysis of the system. The definitions that follow refer to MIMO systems in the form of Eq. 1 .

We will start by reviewing the definition of the relative order of an output variable with respect to the manipulated input vector.

Definition 1. The relative order of the output $y_{i}$ with respect to the manipulated input vector $u, r_{i}$, is defined as the smallest integer for which there exists a $j \in[1, m]$ such that:

$$
L_{g_{j}} L_{f}^{r_{i}-1} h_{i}(x) \neq 0 .
$$

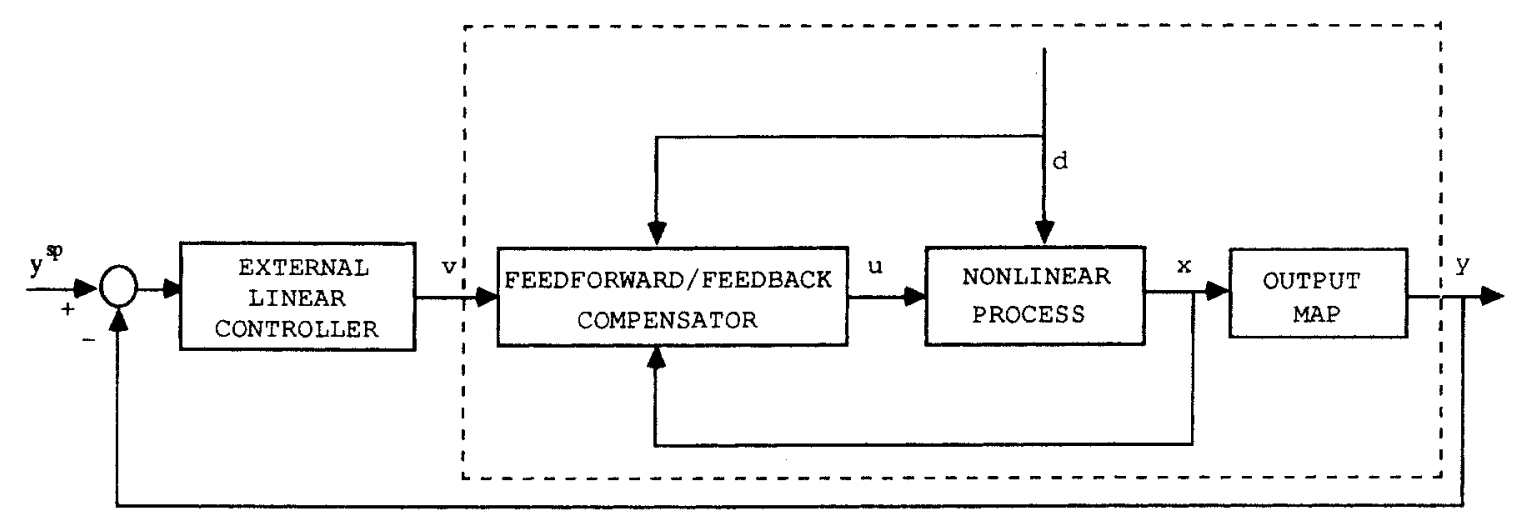

Figure 1. Feedforward/state feedback control structure. 
Equivalently, $r_{i}$ is the smallest integer for which:

$\left[L_{g_{1}} L_{f}^{r_{i}-1} h_{i}(x) L_{g_{2}} L_{f}^{r_{i}-1} h_{i}(x) \cdots L_{g_{m}} L_{f}^{r_{1}-1} h_{i}(x)\right] \neq$ [0 $\left.0 \cdots 0\right]$.

The above concept has been extensively used in multivariable systems theory, with a variety of names depending on the context (e.g., Hirschorn, 1979; $\mathrm{Ha}$ and Gilbert, 1986). It can be easily shown (Kravaris and Soroush, 1990) that $r_{i}$ is the smallest order of derivative of the output $y_{i}$ that explicitly depends on the vector $u$. In this sense, the above concept of relative order characterizes the dynamic effect of the manipulated input vector on each process output. It is therefore extremely meaningful in a multivariable control context, where we are interested in the effect of the manipulated input vector, rather than the effect of each individual manipulated input, on the process outputs. Clearly, in any well-formulated control problem, a finite relative order $r_{i}$ must exist for every output $y_{i}$.

We now generalize the concept of relative order of an output variable with respect to a disturbance input variable, originally introduced for SISO systems by Daoutidis and Kravaris (1989a), in a multivariable setting:

Definition 2. The relative order of the output $y_{i}$ with respect to the disturbance input $d_{k}, \rho_{i x}$, is defined as the smallest integer for which:

$$
L_{w_{x}} L_{f}^{p_{i x}-1} h_{i}(x) \neq 0 .
$$

Note that each disturbance input is treated individually in definition 2. The reason for this is that in a multivariable control context we are concerned with the effect of each individual disturbance on each individual output. It can be easily shown that a finite relative order $\rho_{i \kappa}$ represents the smallest order of derivative of the output $y_{i}$ that explicitly depends on the disturbance $d_{k}$. In this sense, the above concept of relative order characterizes the dynamic effect between disturbance and output variables; small relative orders imply a direct effect of the disturbances on the outputs, rendering thus the regulation of the process an important control issue.

The above assertion can be made more precise through the following structural characterization of disturbances:

Referring to the nonlinear process described by Eq. 1, we introduce the following partition of the set of disturbance inputs into the classes $\mathcal{A}_{i}, \mathscr{B}_{i}$ and $\mathcal{C}_{i}$, associated with the output $y_{i}$ :

$$
\begin{aligned}
& d_{\kappa} \in \mathcal{A}_{i} \Leftrightarrow \rho_{i \kappa}>r_{i} \\
& d_{\kappa} \in \mathcal{B}_{i} \Leftrightarrow \rho_{i \kappa}=r_{i} \\
& d_{\kappa} \in \mathcal{P}_{i} \Leftrightarrow \rho_{i \kappa}<r_{i}
\end{aligned}
$$

Note that for each output, a different partition of the set of the disturbances will be obtained in general. The above partition captures the relative dynamic interactions between the manipu- lated input vector and the individual disturbance inputs for a particular output. More specifically,

- Disturbances that belong to class $\mathcal{A}_{i}$ have a less direct effect on the output $y_{i}$ than the manipulated input vector.

- Disturbances that belong to class $\mathcal{B}_{i}$ have the same effect on the output $y_{i}$ as the manipulated input vector.

- Disturbances that belong to class $\mathcal{C}_{i}$ have a more direct effect on the output $y_{i}$ than the manipulated input vector.

Based on the intuition that has been obtained from the SISO treatment of the subject (Daoutidis and Kravaris, 1989a), the following properties are expected to hold concerning the nature of the regulatory control problem:

- $\mathcal{B}_{i}=\mathcal{C}_{i}=\varnothing:$ pure feedback compensation will suffice to eliminate the effect of the disturbances on the output $y_{i}$.

- $\mathcal{B}_{i} \neq \varnothing, \mathcal{C}_{i}=\varnothing$ : static feedforward/state feedback compensation will be necessary to eliminate the effect of the disturbances on the output $y_{i}$

- $\mathcal{C}_{i} \neq \varnothing$ : dynamic feedforward/state feedback compensation will be necessary to eliminate the effect of the disturbances on the output $y_{i}$.

The overall control action must compensate for each class of disturbances and for each output in an appropriate way.

Remark 1 . In general, definitions 1 and 2 are valid locally, in a region around a point of interest in state space. Singular points may exist throughout the state space.

Remark 2. The concept of relative order concerns the input/ output behavior of the system; therefore, it is invariant under coordinate change and in a linear setting it is associated with the transfer function description of the system and not with the specific state-space realization.

\section{Synthesis of the Feedforward/State Feedback Control Law and Development of the Control Structure}

The main result of this section, which generalizes a previous result for SISO systems (Daoutidis and Kravaris, 1989a), is summarized in the following theorem.

Theorem 1. Consider the MIMO nonlinear process described by Eq. 1. Let $r_{i}$ 's be the relative orders of each output $y_{i}$ with respect to the manipulated input vector and $\rho_{i \mathrm{x}}$ 's be the relative orders of each output with respect to each disturbance input. Also, consider the partitions of the set of disturbances according to Eq. 4 and assume that the characteristic matrix

$$
C(x)=\left[\begin{array}{ccc}
L_{g_{1}} L_{f}^{r_{1}-1} h_{1}(x) & \cdots & L_{g_{m}} L_{f}^{r^{-1}} h_{1}(x) \\
\cdot & & \cdot \\
\cdot & & \cdot \\
L_{g_{1}} L_{f}^{r_{m}-1} h_{m}(x) & \cdots & L_{g_{m}} L_{f}^{r_{m}-1} h_{m}(x)
\end{array}\right]
$$

is nonsingular. Then, the control law:

$$
\begin{aligned}
u=\left[\sum_{i=1}^{m} \beta_{i r_{i}} L_{g_{1}} L_{f}^{r_{j}-1} h_{i}(x)\right. & \left.\cdots \sum_{i=1}^{m} \beta_{i r_{i}} L_{g_{m}} L_{f}^{r_{j}-1} h_{i}(x)\right]^{-1} \\
& \cdot\left\{v-\sum_{i=1}^{m} \sum_{k=0}^{r_{t}} \beta_{i k} L_{f}^{k} h_{i}(x)-\sum_{i=1}^{m} \sum_{d_{k} \in B_{i}} \beta_{i r_{i}} L_{w_{k}} L_{f}^{r_{i}-1} h_{i}(x) d_{k}-\sum_{i=1}^{m} \sum_{d_{k} \in \mathcal{C}_{i}} \sum_{l=0}^{r_{i}-\rho_{i k}} \sum_{k=\rho_{i k}+l}^{r_{i}} \beta_{i k} \frac{d^{l}}{d t^{l}}\left(L_{w_{k}} L_{f}^{k-l-1} h_{i}(x) d_{k}\right)\right\}
\end{aligned}
$$


- Completely eliminates the effect of the disturbances on the process outputs.

- Induces the linear $v-y$ closed-loop response:

$$
\sum_{i=1}^{m} \sum_{k=0}^{r_{i}} \beta_{i k} \frac{d^{k} y_{i}}{d t^{k}}=v
$$

where $\beta_{i k}=\left[\beta_{i k}^{1} \beta_{i k}^{2} \cdots \beta_{i k}^{m}\right]^{T} \in \mathbb{R}^{m}$ are vectors of adjustable constant parameters with

$$
\operatorname{det}\left[\beta_{1 r_{1}} \beta_{2 r_{2}} \cdots \beta_{m r_{m}}\right] \neq 0 \text {, }
$$

and $v=\left[v_{1} v_{2} \cdots v_{m}\right]^{T} \in \mathbb{R}^{m}$ is a vector of reference inputs.

We are going to omit the detailed proof of the above theorem, because it involves a notationally complicated, but conceptually straightforward, procedure. The procedure of the proof goes through the following steps:

a. Derive explicit expressions for the derivatives of each output $y_{i}$ up to $r_{i}$ th order, in terms of $x, u$ and $d_{k}$ 's.

b. Using these expressions, calculate the sum

$$
\sum_{i=1}^{m} \sum_{k}^{n_{i}} \beta_{i k} \frac{d^{k} y_{i}}{d t^{k}}
$$

in terms of $x, u$ and $d_{x}$ 's.

c. Calculate the expression for the manipulated input vector $u$ that makes the above sum equal to $v$.

The interested reader may refer to the SISO case (Daoutidis and Kravaris, 1989a) for the expressions of the derivatives of the outputs that are used in the calculations. It is worth noting that the whole development of the control law is carried out in an algebraic context, with the differential geometric character being implicitly present through the concept of relative order and the Lie derivative notation.

Remark 3. The nonsingularity assumption for the characteristic matrix $C(x)$ and the condition given by Eq. 8 guarantee that the inverse

$$
\begin{aligned}
& {\left[\sum_{i=1}^{m} \beta_{i r_{i}} L_{g_{1}} L_{f}^{r_{i}-1} h_{i}(x) \cdots \sum_{i=1}^{m} \beta_{i r_{i}} L_{g_{m}} L_{f}^{r_{i}-1} h_{i}(x)\right]^{-1}} \\
& \left.=\left\{\begin{array}{ccc}
{\left[\beta_{1 r_{1}} \cdots \beta_{m r_{m}}\right]} \\
L_{g_{1}} L_{f}^{r_{1}-1} h_{1}(x) & \cdots & L_{g_{m}} L_{f}^{r_{1}-1} h_{1}(x) \\
\cdot & & \cdot \\
\cdot & & \cdot \\
\cdot & & \\
L_{g_{1}} L_{f}^{r_{m}-1} h_{m}(x) & \cdots & L_{g_{m}} L_{f}^{r_{m}-1} h_{m}(x)
\end{array}\right]\right\}^{-1}
\end{aligned}
$$

and consequently the control law are well-defined. The nonsingularity assumption for the matrix $C(x)$ is somewhat restrictive, in the sense that it imposes a structural restriction on the nonlinear system. On the other hand, the majority of practical applications conforms with the above assumption. More sophisticated techniques than the ones presented here have to be employed for those that don't.

Despite the apparent complexity of the control law, a simple structure is present. In particular, it is composed of three distinct parts:

- A pure static state feedback part, which accounts for input/output linearity and eliminates, for each $i$, the effect of the disturbances in $\mathcal{A}_{i}$ on the output $y_{i}$ :

$$
\begin{aligned}
{\left[\sum_{i=1}^{m} \beta_{i r_{i}} L_{g_{1}} L_{f}^{r_{i}-1} h_{i}(x) \cdots \sum_{i=1}^{m} \beta_{i r_{i}} L_{g_{m}} L_{f}^{r_{i}-1} h_{i}(x)\right]^{-i} } \\
\cdot\left\{v-\sum_{i=1}^{m} \sum_{k=0}^{r_{i}} \beta_{i k} L_{f}^{k} h_{i}(x)\right\}
\end{aligned}
$$

- A static feedforward/state feedback part which eliminates, for each $i$, the effect of the disturbances in $\mathcal{B}_{i}$ on the output $y_{i}$ :

$$
\begin{aligned}
{\left[\sum_{i=1}^{m} \beta_{i r_{i}} L_{g_{1}} L_{f}^{r_{i}-1} h_{i}(x) \cdots \sum_{i=1}^{m} \beta_{i r_{i}} L_{g_{m}} L_{f}^{r_{i}-1} h_{i}(x)\right]^{-1} } \\
\cdot\left\{-\sum_{i=1}^{m} \sum_{d_{k} \in \mathcal{B}_{i}} \beta_{i r_{i}} L_{w_{k}} L_{f}^{r_{i}-1} h_{i}(x) d_{\kappa}\right\}
\end{aligned}
$$

- A dynamic feedforward/state feedback part which eliminates, for each $i$, the effect of the disturbances in $\mathcal{C}_{i}$ on the output $y_{i}$ :

$$
\begin{aligned}
{\left[\sum_{i=1}^{m} \beta_{i r_{i}} L_{g_{1}} L_{f}^{r_{i}-1} h_{i}(x) \cdots \sum_{i=1}^{m} \beta_{i r_{i}} L_{g_{m !}} L_{f}^{r_{i}-1} h_{i}(x)\right]^{-1} } \\
\cdot\left\{-\sum_{i=1}^{m} \sum_{d_{k} \in \mathcal{C}_{i}} \sum_{l=0}^{r_{i}-\rho_{i k}} \sum_{k=\rho_{i n}+l}^{r_{i}} \beta_{i k} \frac{d^{l}}{d t^{l}}\left(L_{w_{k}} L_{f}^{k-l-1} h_{i}(x) d_{\mathrm{k}}\right)\right\}
\end{aligned}
$$

The dynamic element in the above part can be implemented using a lead-lag type of approximation of appropriate order.

In each one of the above parts of the control law, the control action results by superimposing explicit compensation terms for each output and for each disturbance, depending on the corresponding partition of the set of disturbances. It is exactly this explicit character of the control law that results in its rather complicated form. More compact expressions can be written, by adopting a more compact vector notation.

At this point, let us summarize the basic characteristics of the proposed control law. By

- Calculating the relative orders $r_{i}$ and $\rho_{i \kappa}$ for every output $y_{i}$

- Implementing the control law given by $\mathrm{Eq} .6$ for an appropriate choice of the adjustable parameters $\beta_{i k}^{j}$ 's we use all the available process information so that:

- The input/output behavior of the closed-loop system for changes in the reference inputs is linear

- The regulatory behavior of the closed-loop system is perfect with respect to the measurable disturbances under the assumption, of course, of a perfect model and perfect implementation of the controller. In the next section, we will associate the choice of the adjustable parameters $\beta_{i k}^{j}$ 's with the stability characteristics and the degree of coupling in the closed-loop system. It should be mentioned that an implicit assumption in the previous development is that measurements of the system's states are available. In fact, this assumption is a key one in obtaining the perfect disturbance rejection property on the output $y_{i}$ for the disturbances that belong to the class $\mathcal{A}_{i}$, without using measurements of these disturbances. The measurements of the states completely capture the effect of these disturbances and since the manipulated input vector has a more 
direct effect on the output $y_{i}$ than these disturbances, it completely compensates for their effect.

\section{Simplifications and generalizations}

The control law given by Eq. 6 simplifies greatly in the following two cases:

$$
\begin{aligned}
& \bullet \mathcal{C}_{i}=\varnothing \text { for every } i \text { : } \\
& u=\left[\sum_{i=1}^{m} \beta_{i r_{i}} L_{g_{1}} L_{f}^{r_{i}-1} h_{i}(x) \cdots \sum_{i=1}^{m} \beta_{i r_{i}} L_{g_{m}} L_{f}^{r_{i}-1} h_{i}(x)\right]^{-1} \\
& \qquad\left\{v-\sum_{i=1}^{m} \sum_{k=0}^{r_{i}} \beta_{i k} L_{f}^{k} h_{i}(x)-\sum_{i=1}^{m} \sum_{d, \in \mathcal{B}_{i}} \beta_{i r_{i}} L_{w_{k}} L_{f}^{r_{i}-1} h_{i}(x) d_{k}\right\}
\end{aligned}
$$

which is a static feedforward/state feedback control law.

$$
\text { - } \mathcal{B}_{i}=\mathcal{C}_{i}=\varnothing \text { for every } i
$$

$$
\begin{aligned}
u=\left[\sum_{i=1}^{m} \beta_{i r_{i}} L_{g_{1}} L_{f}^{r_{i}-1} h_{i}(x) \cdots \sum_{i=1}^{m} \beta_{i r_{i}} L_{g_{m}} L_{f}^{r_{i}-1} h_{i}(x)\right]^{-1} \\
\cdot\left\{v-\sum_{i=1}^{m} \sum_{k=0}^{r_{i}} \beta_{i k} L_{f}^{k} h_{i}(x)\right\}
\end{aligned}
$$

which is a pure state feedback control law, exactly the same as if no disturbances were present (Kravaris and Soroush, 1990).

The above results conform with the intuitive arguments of the previous section concerning the nature of the control law, depending on the nonvoid classes of disturbances present in each partition.

In the case of $m=1$ (SISO system), the control law reduces to:

$$
u=\left\{\beta_{r} L_{g} L_{f}^{r-1} h(x)\right\}^{-1}\left\{v-\sum_{k=0}^{r} \beta_{k} L_{f}^{k} h(x)-\sum_{d_{k} \in \mathcal{B}} \beta_{r} L_{w_{k}} L_{f}^{r-1} h(x) d_{k}-\sum_{d_{k} \in \mathcal{C}} \sum_{l=0}^{r-\rho_{k}} \sum_{k=\rho_{k}+l}^{r} \beta_{k} \frac{d^{l}}{d t^{l}}\left(L_{w_{k}} L_{f}^{k-l-1} h(x) d_{k}\right)\right\}
$$

which, as expected, is exactly the same with our previous result for SISO systems (Daoutidis and Kravaris, 1989a).

The nonsingularity of the characteristic matrix $C(x)$ (Eq. 5) is a sufficient condition for a static state feedback input/output linearizing control law to exist. It has been shown (Kravaris and Soroush, 1990), however, that input/output linearization can be achieved for a larger class of disturbance-free systems than those satisfying this assumption. Generalization of theorem 1 for this class of systems is possible, but it would require complicated notation and several technicalities that would dilute the focus of the present paper.

The proposed methodology can also be trivially generalized to achieve any nonlinear input/output closed-loop response of the form:

$$
\Phi\left(y_{1}, \frac{d y_{1}}{d t}, \ldots, \frac{d^{r_{1}} y_{1}}{d t^{r_{1}}}, \ldots, y_{m}, \frac{d y_{m}}{d t}, \ldots, \frac{d^{r_{m}} y_{m}}{d t^{r_{m}}}\right)=v
$$

Finally, consider the more general class of nonlinear processes with a state-space description of the form:

$$
\begin{aligned}
& \dot{x}=f(x)+\sum_{j=1}^{m} g_{j}(x) \phi_{j}\left(x, u_{j}, d_{j}^{*}\right)+\sum_{k=1}^{p} w_{k}(x) d_{k} \\
& y_{i}=h_{i}(x), i=1, \ldots, m
\end{aligned}
$$

where $\phi_{j}\left(x, u_{j}, d_{j}^{*}\right)$ is a scalar function solvable for $u_{j}$, and $d_{j}^{*}$ is a vector of additional measurable disturbances. The above class incorporates cases where some manipulated inputs appear in the state equations coupled with some measurable disturbances. In this case, the proposed methodology can be applied by simply letting $U_{j}=\phi_{j}\left(x, u_{j}, d_{j}^{*}\right)$, calculating the control law (Eq. 6) for the new manipulated inputs $U_{j}$ and then solving for the original manipulated inputs $u_{j}$.

Remark 4. The relation of the feedforward/feedback problem to the classical disturbance decoupling problem was discussed for SISO systems in Daoutidis and Kravaris (1989b). It was shown that our proposed feedforward/feedback formulation applies to a much more general class of systems than disturbance decoupling and gives as a byproduct a much simpler and more transparent necessary and sufficient condition for solvability of the disturbance decoupling problem.

In the context of MIMO systems of the form given by Eq. 1 and assuming that the characteristic matrix $C(x)$ is nonsingular, the necessary and sufficient condition found in the literature (e.g., Isidori et al., 1981) for solvability of the disturbance decoupling problem is:

$$
\begin{aligned}
w_{k}(x) \in \bigcap_{i=1}^{m}\left[\operatorname{ker}\left\{d h_{i}(x)\right\} \cap \operatorname{ker}\left\{d L_{f} h_{i}(x)\right\}\right. \\
\left.\qquad \cdots \cap \operatorname{ker}\left\{d L_{f}^{r_{i}-1} h_{i}(x)\right\}\right]
\end{aligned}
$$

for every $x \in \mathbb{R}^{n}$ and every $k$.

Within our proposed feedforward/feedback formulation, the above condition can be easily shown to be equivalent to:

$$
\rho_{i \kappa}<r_{i} \Leftrightarrow d_{\kappa} \in \mathcal{A}_{i}
$$

for every $i$ and $\kappa$, which is much simpler and easier to verify than Eq. 9.

Clearly, the above condition is extremely restrictive and is rarely met in real systems. On the other hand, our proposed methodology provides a systematic way of treating measurable disturbances, allowing for them to be incorporated in the control law in an appropriate way.

Remark 5. The disturbance rejection capability of the proposed methodology with respect to the disturbances in class $\mathcal{A}_{i}$ can find an interesting robustness interpretation. In particular, consider a localized perturbation (model uncertainty and/or unmeasured disturbance) of arbitrary magnitude which enters the system dynamic structure in an additive way at a certain location (i.e., a certain state equation). Such a perturbation can be viewed as an unmeasurable disturbance and can be assigned an "equivalent relative order." The particular perturbation can then be included in one of the classes of disturbances defined by Eq. 4 . In case it belongs to the class $\mathcal{A}_{i}$, it will not have any 


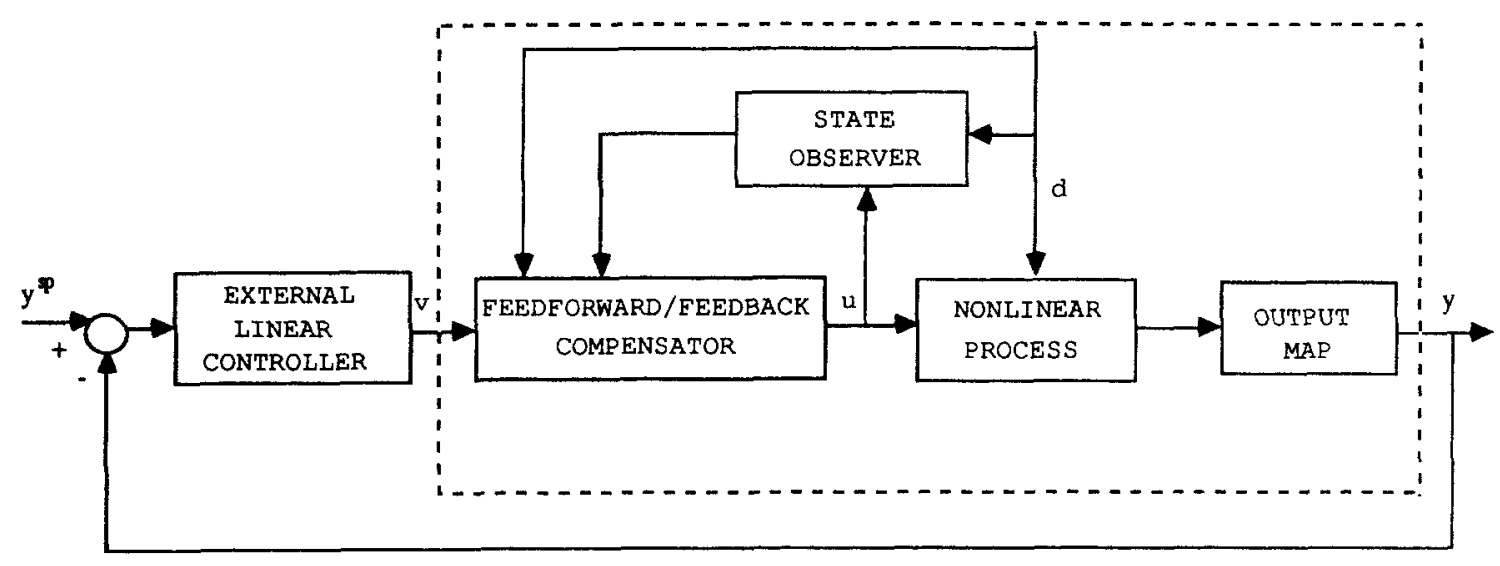

Figure 2. Feedforward/output feedback control structure.

effect on the output $y_{i}$ in the closed-loop system. The above inherent robustness feature of our methodology is extremely meaningful in chemical systems, where a model uncertainty can often be identified with errors in certain system parameters, e.g., kinetic rate constants and heat transfer coefficients.

\section{Feedforward/feedback control structures}

Theorem 1 provides an explicit solution to the synthesis problem posed in the introduction. In general, as was discussed, the control structure has to be completed by a linear MIMO controller with integral action, designed appropriately to account for unmeasured disturbances and/or model uncertainty. The resulting overall feedforward/state feedback control structure is depicted in Figure 1. In the case where the disturbances are not modeled explicitly and therefore are not used in the control law, the above control structure reduces to the MIMO GLC control structure (Kravaris and Soroush, 1990).

In many practical situations, on-line measurements of the states are not available or economically justified. In this case and for an open-loop asymptotically stable system, a simple method of reconstructing the states is the use of an open-loop state observer. In particular, given estimates of the initial states $\hat{x}(0)=\hat{x}_{0}$, and driven by on-line measurements of the process inputs (manipulated and disturbance), a replica of the process model

$$
\dot{\hat{x}}=f(\hat{x})+\sum_{j=1}^{m} g_{j}(\hat{x}) u_{j}+\sum_{k=1}^{p} w_{k}(\hat{x}) d_{k}
$$

will converge to the correct estimates of the states for large $t$; any initialization error will eventually die out. The resulting control structure after incorporating the open-loop state observer becomes a feedforward/output feedback control structure, as it is shown in Figure 2. Obviously, in such a control structure one has to use measurements of all the disturbances, even the ones that belong to the classes $\mathcal{A}_{i}$, since there is some lost information from the states that are not measured.

\section{Closed-Loop Design Considerations}

As indicated by the control structure, the proposed control methodology is a two-step procedure that involves: a) the design of the inner feedforward/feedback loop ( $v-y$ system); and b) the design of a multivariable external linear controller to ensure satisfactory regulatory behavior of the closed-loop system in the presence of unmeasurable disturbances and model uncertainty.

\section{Design of the feedforward/feedback inner loop}

Under the control law given by Eq. 6, the dynamics of the $v-$ $y$ system is governed by:

$$
\begin{aligned}
\left(\beta_{10} y_{1}+\cdots+\beta_{1 r_{1}}\right. & \left.\frac{d^{r_{1}} y_{1}}{d t^{r_{1}}}\right)+\cdots \\
& +\left(\beta_{m 0} y_{m}+\cdots+\beta_{m r_{m}} \frac{d^{r_{m}} y_{m}}{d t^{r_{m}}}\right)=v
\end{aligned}
$$

or expanding the $\beta$-column notation:

$$
\begin{gathered}
\left(\beta_{10}^{\mathrm{l}} y_{1}+\cdots+\beta_{1 r_{1}}^{1} \frac{d^{r_{1}} y_{1}}{d t^{r_{1}}}\right)+\cdots+\left(\beta_{m 0}^{1} y_{m}+\cdots+\beta_{m r_{m}}^{1} \frac{d^{r_{m}} y_{m}}{d t^{r_{m}}}\right)=v_{1} \\
\left(\beta_{10}^{2} y_{1}+\cdots+\beta_{1 r_{1}}^{2} \frac{d^{r^{\prime}} y_{1}}{d t^{r_{1}}}\right)+\cdots+\left(\beta_{m 0}^{2} y_{m}+\cdots+\beta_{m r_{m}}^{2} \frac{d^{r_{m}} y_{m}}{d t^{r_{m}}}\right)=v_{2} \\
\vdots \\
\vdots \\
\left(\beta_{10}^{m} y_{1}+\cdots+\beta_{1 r_{1}}^{m} \frac{d^{\prime} y_{1}}{d t^{r_{1}}}\right)+\cdots+\left(\beta_{m 0}^{m} y_{m}+\cdots+\beta_{m r_{m}}^{m} \frac{d^{r_{m}} y_{m}}{d t^{m_{m}}}\right)=v_{m}
\end{gathered}
$$


or in the Laplace domain and using a matrix fraction description:

$$
y(s)=\left[\left(\sum_{k=0}^{r_{1}} \beta_{1 k} s^{k}\right)\left(\sum_{k=0}^{r_{2}} \beta_{2 k} s^{k}\right) \cdots\left(\sum_{k=0}^{r_{m}} \beta_{m k} s^{k}\right)\right]^{-1} v(s)
$$

The order of the closed-loop system is $r_{1}+r_{2}+\cdots+r_{m}$. In analogy with the SISO case, the closed-loop system does not possess any finite zeros. On the other hand, the poles of the closed-loop system are the roots of the characteristic equation:

$$
\operatorname{det}\left[\left(\sum_{k=0}^{r_{1}} \beta_{1 k} s^{k}\right)\left(\sum_{k=0}^{r_{2}} \beta_{2 k} s^{k}\right) \cdots\left(\sum_{k=0}^{r_{m}} \beta_{m k} s^{k}\right)\right]=0 .
$$

Consequently, the BIBO stability characteristics of the closedloop system depend on the values of the $m\left(r_{1}+\cdots+r_{m}\right)+$ $m^{2}$ adjustable parameters $\beta_{i k}^{j}$ 's.

The issue of closed-loop asymptotic stability of the states under no external inputs is not going to be addressed in detail in this work, for brevity. The procedure to be followed in order to obtain a precise characterization for this issue is conceptually similar to the one followed in the SISO case (Daoutidis and Kravaris, 1989a), but the treatment must be done in a MIMO setting using a multivariable notion of nonlinear zeros. In particular, one can generalize the disturbance-free concept of MIMO zero dynamics and obtain a concept of zero dynamics for MIMO nonlinear systems with disturbances; then, appropriate stability conditions on the zero dynamics will guarantee the asymptotic stability of the unforced closed-loop system.

In some cases, it may be desirable to achieve input/output decoupling in the closed-loop system, i.e., to have each reference input $v_{i}$ affect only the output $y_{i}$. In this case, the postulated closed-loop response is:

$$
\begin{gathered}
\left(\beta_{10}^{1} y_{1}+\cdots+\beta_{1 r_{1}}^{1} \frac{d^{r_{1}} y_{1}}{d t^{r_{1}}}\right)=v_{1} \\
\left(\beta_{20}^{2} y_{2}+\cdots+\beta_{2 r_{2}}^{2} \frac{d^{r_{2}} y_{2}}{d t^{r_{2}}}\right)=v_{2} \\
\cdot \\
\cdot \\
\left(\beta_{m 0}^{m} y_{m}+\cdots+\beta_{m r_{m}}^{m} \frac{d^{r^{m}} y_{m}}{d t^{r_{m}}}\right)=v_{m}
\end{gathered}
$$

and one simply sets

$$
\beta_{i k}^{j}=0, i \neq j
$$

in the control law.

Any kind of partially decoupled closed-loop response can also be achieved by appropriate choice of the adjustable parameters $\beta_{i k}^{j}$ 's, as long as the nonsingularity condition of Eq. 8 is satisfied. By requesting any kind of input/output decoupling, we may impose an additional structural constraint on the closedloop system which may cause deterioration in its performance characteristics. On the other hand, several advantages are present, such as fewer adjustable parameters $\left[\left(r_{1}+\cdots+r_{m}+m\right)\right.$, in the case of full decoupling $]$ and the use of SISO controllers in the external loop, in which case their tuning is straightforward. Physical constraints on the manipulated inputs and/or physical importance of the controlled outputs may often dictate whether decoupling is realistic and/or desirable. In general, despite the extensive research effort in this area, there is a lack of systematic methods of fundamental rigor for assessing when decoupling is favorable, even in the case of linear systems. It should be noted that, within our proposed synthesis framework, any degree of coupling can be achieved by simply an appropriate choice of the adjustable parameters (without any modification in the actual synthesis procedure). This fact allows a significant degree of flexibility to the designer, who can incorporate his/her own intuition and experience in the tuning procedure and test the resulting performance characteristics.

\section{Design of the external linear controller}

The design of a multivariable linear controller for the linear $v-y$ system can be performed using techniques from linear control theory. Of course, if the $v-y$ system is decoupled, the designer has a much simpler task of synthesizing and tuning of the corresponding SISO linear controllers. In any case, the external linear controller must be designed to ensure

- Stability of the overall closed-loop system $y^{s p}-y$

- Satisfactory tracking of set points and rejection of the unmeasurable disturbances

- That the magnitude of the manipulated inputs $u_{j}$ 's does not exceed the bounds imposed by practical constraints.

\section{Application of the Feedforward/Feedback Control Methodology to a Continuous Polymerization Reactor}

Consider the CSTR shown in Figure 3, where free-radical polymerization of methyl methacrylate (MMA) takes place, with azo-bis-isobutyronitrile (AIBN) as initiator and toluene as solvent. The reaction is exothermic and a cooling jacket allows the heat removal. The standard mechanism of free-radical polymerization is assumed, together with the resulting rate laws (Ray, 1972; Ray et al., 1971; Congalidis et al., 1989; Schmidt and Ray, 1981; Tsoukas et al., 1982). We also make the following assumptions:

- Perfect mixing in the reactor

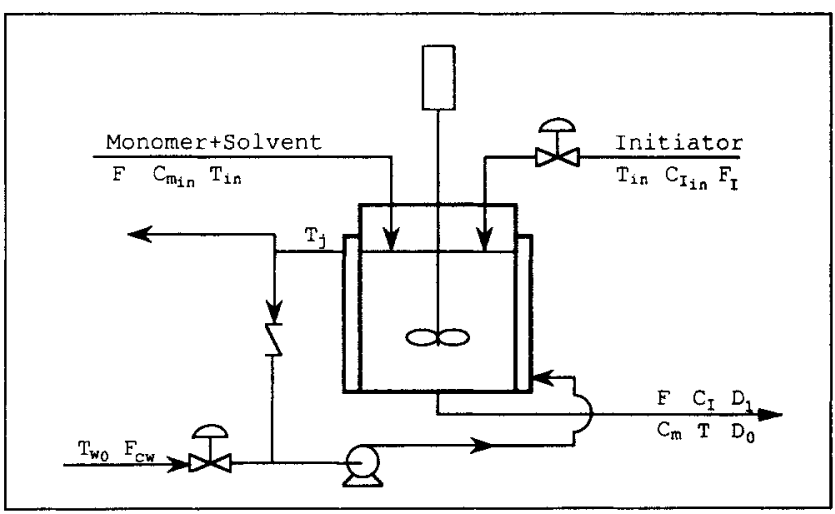

Figure 3. Simulated continuous polymerization reactor. 
- Constant density of the reacting mixture (no volume shrinkage)

- Constant heat capacity of the reacting mixture

- Uniform coolant temperature in the jacket

- Insulated reactor and cooling system

- Constant density and heat capacity of the coolant

- No polymer in the inlet streams

- No gel effect (because of low monomer conversion)
- Constant reactor volume (constant volumetric flow rate of the monomer stream)

- Negligible flow rate of the initiator solution compared to that of the monomer stream

- Negligible inhibition and chain transfer to solvent reactions

- Quasisteady state and long-chain hypothesis.

The dynamic behavior of the process is then described by the following mass and energy balances:

$$
\begin{aligned}
\frac{d C_{m}}{d t} & =-\left[Z_{p} \exp \left(\frac{-E_{p}}{R T}\right)+Z_{f_{m}} \exp \left(\frac{-E_{f_{m}}}{R T}\right)\right] C_{m} P_{0}\left(C_{l}, T\right)+\frac{F\left(C_{m_{i x}}-C_{m}\right)}{V} \\
\frac{d C_{l}}{d t} & =-Z_{I} \exp \left(\frac{-E_{l}}{R T}\right) C_{I}+\frac{F_{l} C_{I_{\text {in }}}-F C_{I}}{V} \\
\frac{d T}{d t} & =Z_{p} \exp \left(\frac{-E_{p}}{R T}\right) C_{m} \frac{\left(-\Delta H_{P}\right)}{\rho c_{p}} P_{0}\left(C_{l}, T\right)-\frac{U A}{\rho c_{p} V}\left(T-T_{j}\right)+\frac{F\left(T_{i n}-T\right)}{V} \\
\frac{d D_{0}}{d t} & =\left[0.5 Z_{T_{r}} \exp \left(\frac{-E_{T_{c}}}{R T}\right)+Z_{T_{d}} \exp \left(\frac{-E_{T_{d}}}{R T}\right)\right]\left[P_{0}\left(C_{I}, T\right)\right]^{2}+Z_{f_{m}} \exp \left(\frac{-E_{f_{m}}}{R T}\right) C_{m} P_{0}\left(C_{I}, T\right)-\frac{F D_{0}}{V} \\
\frac{d D_{1}}{d t} & =M_{m}\left[Z_{p} \exp \left(\frac{-E_{p}}{R T}\right)+Z_{f_{m}} \exp \left(\frac{-E_{f_{m}}}{R T}\right)\right] C_{m} P_{0}\left(C_{l}, T\right)-\frac{F D_{1}}{V} \\
\frac{d T_{j}}{d t} & =\frac{F_{c w}}{V_{o}}\left(T_{w_{o}}-T_{j}\right)+\frac{U A}{\rho_{w} c_{w} V_{o}}\left(T-T_{j}\right)
\end{aligned}
$$

where

$$
P_{0}\left(C_{l}, T\right)=\left[\frac{2 f^{*} C_{l} Z_{l} \exp \left(\frac{-E_{l}}{R T}\right)}{Z_{T_{d}} \exp \left(\frac{-E_{T_{d}}}{R T}\right)+Z_{T_{c}} \exp \left(\frac{-E_{T_{r}}}{R T}\right)}\right]^{0.5}
$$

Control of the temperature $T$ and the number average molecular weight $D_{1} / D_{0}$ of the polymer product is considered, by manipulating the volumetric flow rate of the initiator $F_{1}$ and the volumetric flow rate of the cooling water $F_{c w}$. The concentration of monomer in the inlet stream $C_{m_{i n}}$ and the temperature of the inlet stream $T_{i n}$ are the major measurable disturbances. Thus, following the standard procedure and letting

$$
x_{1}=C_{m}, x_{2}=C_{l}, x_{3}=T, x_{4}=D_{0}, x_{5}=D_{1}, x_{6}=T_{j}
$$

and

$$
\begin{gathered}
u_{1}=F_{l}, u_{2}=F_{c w} \\
d_{1}=C_{m_{i n}}, d_{2}=T_{i n} \\
y_{1}=\frac{D_{1}}{D_{0}}, y_{2}=T
\end{gathered}
$$

the dynamic equations of the process are put in the form of Eq. 1 , where $n=6, m=2, p=2$ and

$$
f(x)=\left[\begin{array}{c}
f_{1}(x) \\
f_{2}(x) \\
f_{3}(x) \\
f_{4}(x) \\
f_{5}(x) \\
f_{6}(x)
\end{array}\right]=\left[\begin{array}{c}
-\left[Z_{p} \exp \left(\frac{-E_{p}}{R x_{3}}\right)+Z_{f_{m}} \exp \left(\frac{-E_{f_{m}}}{R x_{3}}\right)\right] x_{1} P_{0}\left(x_{2}, x_{3}\right)-\frac{F x_{1}}{V} \\
-Z_{I} \exp \left(\frac{-E_{l}}{R x_{3}}\right) x_{2}-\frac{F x_{2}}{V} \\
Z_{p} \exp \left(\frac{-E_{p}}{R x_{3}}\right) x_{1} \frac{\left(-\Delta H_{P}\right)}{\rho c_{p}} P_{0}\left(x_{2}, x_{3}\right)-\frac{U A}{\rho c_{p} V}\left(x_{3}-x_{6}\right)-\frac{F x_{3}}{V} \\
\left.0.5 Z_{T_{c}} \exp \left(\frac{-E_{T_{c}}}{R x_{3}}\right)+Z_{T_{d}} \exp \left(\frac{-E_{T_{f}}}{R x_{3}}\right)\right]\left[P_{0}\left(x_{2}, x_{3}\right)\right]^{2}+Z_{f_{m}} \exp \left(\frac{-E_{f_{m}}}{R x_{3}}\right) x_{1} P_{0}\left(x_{2}, x_{3}\right)-\frac{F x_{4}}{V} \\
M_{m}\left[Z_{p} \exp \left(\frac{-E_{p}}{R x_{3}}\right)+Z_{f_{m}} \exp \left(\frac{-E_{f_{m}}}{R x_{3}}\right)\right] x_{1} P_{0}\left(x_{2}, x_{3}\right)-\frac{F x_{5}}{V} \\
\frac{U A}{\rho_{w} c_{w} V_{o}}\left(x_{3}-x_{6}\right)
\end{array}\right]
$$




$$
\begin{gathered}
g_{1}(x)=\left[\begin{array}{c}
0 \\
\frac{C_{I_{i n}}}{V} \\
0 \\
0 \\
0 \\
0
\end{array}\right], g_{2}(x)=\left[\begin{array}{c}
0 \\
0 \\
0 \\
0 \\
0 \\
\frac{T_{w_{0}}-x_{6}}{V_{0}}
\end{array}\right], w_{1}(x)=\left[\begin{array}{c}
F \\
V \\
0 \\
0 \\
0 \\
0
\end{array}\right], w_{2}(x)=\left[\begin{array}{c}
0 \\
0 \\
\frac{F}{V} \\
0 \\
0 \\
0
\end{array}\right] \\
h_{1}(x)=\frac{x_{5}}{x_{4}}, h_{2}(x)=x_{3}
\end{gathered}
$$

Based on definitions 1 and 2, the relative orders are easily found to take the following values:

- Output $y_{1}: r_{1}=2, \rho_{11}=2, \rho_{12}=2$

- Output $y_{2}: r_{2}=2, \rho_{21}=2, \rho_{22}=1$

Consequently, the set of disturbances is partitioned as follows:

- $\mathcal{A}_{1}=\varnothing, \mathscr{B}_{1}=\left\{d_{1}, d_{2}\right\}, e_{1}=\varnothing$

- $\mathcal{A}_{2}=\varnothing, \mathscr{B}_{2}=\left\{d_{1}\right\}, \mathcal{C}_{2}=\left\{d_{2}\right\}$

Clearly, as a result of the dynamic structure of the particular process, we will need static feedforward/feedback compensation to eliminate the effect of $d_{1}$ and $d_{2}$ on $y_{1}$ and the effect of $d_{1}$ on $y_{2}$, while dynamic feedforward/feedback compensation will be necessary to eliminate the effect of $d_{2}$ on $y_{2}$.

The characteristic matrix $C(x)$ of this system defined by Eq. 5 becomes:

$$
C(x)=\left[\begin{array}{ll}
L_{g_{1}} L_{f} h_{1}(x) & L_{g_{2}} L_{f} h_{1}(x) \\
L_{g_{1}} L_{f} h_{2}(x) & L_{g_{2}} L_{f} h_{2}(x)
\end{array}\right]
$$

where

$$
\begin{aligned}
& L_{g_{1}} L_{f} h_{1}(x)=\left[\frac{\partial f_{5}(x)}{\partial x_{2}}-\frac{x_{9}}{x_{4}} \frac{\partial f_{4}(x)}{\partial x_{2}}\right] \frac{C_{l_{i n}}}{V x_{4}} \\
& L_{g_{2}} L_{f} h_{1}(x)=0 \\
& L_{g_{1}} L_{f} h_{2}(x)=\frac{\partial f_{3}(x)}{\partial x_{2}} \frac{C_{l_{i n}}}{V} \\
& L_{g_{2}} L_{f} h_{2}(x)=\frac{U A}{\rho c_{p} V} \frac{T_{w_{o}}-x_{6}}{V_{o}}
\end{aligned}
$$

It can be easily checked that $C(x)$ is generically nonsingular; the feedforward/state feedback control law given by Eq. 6 can therefore be applied in a straightforward fashion, yielding:

$$
\begin{aligned}
u=\left[\beta_{12} L_{g_{1}} L_{f} h_{1}(x)+\right. & \left.\beta_{22} L_{g_{1}} L_{f} h_{2}(x) \quad \beta_{12} L_{g_{2}} L_{f} h_{1}(x)+\beta_{22} L_{g_{2}} L_{f} h_{2}(x)\right]^{-1} \\
& \cdot\left\{v-\sum_{i=1}^{2} \sum_{k=0}^{2} \beta_{i k} L_{f}^{k} h_{i}(x)-\left(\beta_{12} L_{w_{1}} L_{f} h_{1}(x) d_{1}+\beta_{12} L_{w_{2}} L_{f} h_{1}(x) d_{2}+\beta_{22} L_{w_{1}} L_{f} h_{2}(x) d_{1}\right)\right. \\
& \left.-\left(\beta_{21} L_{w_{2}} h_{2}(x) d_{2}+\beta_{22} L_{w_{2}} L_{f} h_{2}(x) d_{2}+\beta_{22} \frac{d}{d t}\left(L_{w_{2}} h_{2}(x) d_{2}\right)\right)\right\}
\end{aligned}
$$

where

$$
\begin{aligned}
L_{f} h_{1}(x)= & {\left[f_{5}(x)-\frac{x_{5}}{x_{4}} f_{4}(x)\right] \frac{1}{x_{4}} } \\
L_{f} h_{2}(x)= & f_{3}(x) \\
L_{f}^{2} h_{1}(x)= & \frac{f_{1}(x)}{x_{4}}\left[\frac{\partial f_{5}(x)}{\partial x_{1}}-\frac{x_{5}}{x_{4}} \frac{\partial f_{4}(x)}{\partial x_{1}}\right]+\frac{f_{2}(x)}{x_{4}}\left[\frac{\partial f_{5}(x)}{\partial x_{2}}-\frac{x_{5}}{x_{4}} \frac{\partial f_{4}(x)}{\partial x_{2}}\right] \\
& +\frac{f_{3}(x)}{x_{4}}\left[\frac{\partial f_{5}(x)}{\partial x_{3}}-\frac{x_{5}}{x_{4}} \frac{\partial f_{4}(x)}{\partial x_{3}}\right]+\frac{f_{4}(x)}{x_{4}^{2}}\left[\frac{x_{5} F}{V}-f_{5}(x)+\frac{2 x_{5} f_{4}(x)}{x_{4}}\right]+\frac{f_{5}(x)}{x_{4}}\left[\frac{-F}{V}-\frac{f_{4}(x)}{x_{4}}\right] \\
L_{f}^{2} h_{2}(x)= & \frac{\partial f_{3}(x)}{\partial x_{1}} f_{1}(x)+\frac{\partial f_{3}(x)}{\partial x_{2}} f_{2}(x)+\frac{\partial f_{3}(x)}{\partial x_{3}} f_{3}(x)+\frac{\partial f_{3}(x)}{\partial x_{6}} f_{6}(x) \\
L_{w_{2}} h_{2}(x)= & \frac{F}{V}
\end{aligned}
$$




$$
\begin{aligned}
& L_{w_{1}} L_{f} h_{1}(x)=\left[\frac{\partial f_{5}(x)}{\partial x_{1}}-\frac{x_{5}}{x_{4}} \frac{\partial f_{4}(x)}{\partial x_{1}}\right] \frac{F}{V x_{4}} \\
& L_{w_{2}} L_{f} h_{1}(x)=\left[\frac{\partial f_{5}(x)}{\partial x_{3}}-\frac{x_{5}}{x_{4}} \frac{\partial f_{4}(x)}{\partial x_{3}}\right] \frac{F}{V x_{4}} \\
& L_{w_{1}} L_{f} h_{2}(x)=\frac{\partial f_{3}(x)}{\partial x_{1}} \frac{F}{V} \\
& L_{w_{2}} L_{f} h_{2}(x)=\frac{\partial f_{3}(x)}{\partial x_{3}} \frac{F}{V}
\end{aligned}
$$

The control law given by Eq. 16 has a static feedforward/state feedback component and a dynamic feedforward/state feedback component; this agrees with the intuitive arguments made earlier. In order to obtain full input/output decoupling of the form

$$
\left\{\begin{array}{l}
y_{1}+\beta_{11}^{1} \frac{d y_{1}}{d t}+\beta_{12}^{1} \frac{d^{2} y_{1}}{d t^{2}}=v_{1} \\
y_{2}+\beta_{21}^{2} \frac{d y_{2}}{d t}+\beta_{22}^{2} \frac{d^{2} y_{2}}{d t^{2}}=v_{2}
\end{array}\right.
$$

we simply let

$$
\begin{aligned}
& \beta_{20}^{1}=\beta_{21}^{1}=\beta_{22}^{1}=0, \beta_{20}^{2}=1 \\
& \beta_{10}^{2}=\beta_{11}^{2}=\beta_{12}^{2}=0, \beta_{10}^{1}=1
\end{aligned}
$$

in the control law (Eq. 16), which takes the form

$$
\begin{aligned}
{\left[\begin{array}{l}
u_{1} \\
u_{2}
\end{array}\right]=} & {\left[\begin{array}{cc}
\beta_{12}^{1} L_{g_{1}} L_{f} h_{1}(x) & 0 \\
\beta_{22}^{2} L_{g_{1}} L_{f} h_{2}(x) & \beta_{22}^{2} L_{g_{2}} L_{f} h_{2}(x)
\end{array}\right]^{-1}\left\{\left[\begin{array}{l}
v_{1} \\
v_{2}
\end{array}\right]\right.} \\
& -\left[\begin{array}{c}
h_{1}(x)+\beta_{11}^{1} L_{f} h_{1}(x)+\beta_{12}^{1}\left(L_{f}^{2} h_{1}(x)+L_{w_{1}} L_{f} h_{1}(x) d_{1}+L_{w_{2}} L_{f} h_{1}(x) d_{2}\right) \\
\left.\left.h_{2}(x)+\beta_{21}^{2}\left(L_{f} h_{2}(x)+L_{w_{2}} h_{2}(x) d_{2}\right)+\beta_{22}^{2}\left(L_{f}^{2} h_{2}(x)+L_{w_{1}} L_{f} h_{2}(x) d_{1}+L_{w_{2}} L_{f} h_{2}(x) d_{2}+\frac{d}{d t}\left(L_{w_{2}} h_{2}(x) d_{2}\right)\right)\right]\right\}
\end{array}\right.
\end{aligned}
$$

The kinetic and physical parameters and the operating steady state conditions for the particular process are given in Tables 1 and 2. Integration of the system dynamic equations was performed (after appropriate dedimensionalization) by using the subroutine LSODA from the ODEBACK Library, on the Apollo network of The University of Michigan. The values for the adjustable parameters in the FF/FB control law (Eq. 17) were chosen as

$$
\beta_{11}^{1}=\beta_{21}^{2}=0.44 h, \beta_{12}^{1}=\beta_{22}^{2}=0.016 h^{2}
$$

to place the closed-loop poles at -2.5 and -25.0 for the two decoupled $v_{1}-y_{1}$ and $v_{2}-y_{2}$ systems. The location of the closed-loop poles was chosen so that the constraints on the magni- tude of the manipulated input variables $\left(F_{I} \geq 0\right.$ and $\left.F_{c w} \geq 0\right)$ be satisfied. The external linear controllers in the FF/FB control structure were chosen as two PI controllers with settings $K_{c}=$ 15 and $\tau_{I}=0.4 \mathrm{~h}$. A number of simulation runs verified the stability of the open-loop system around the operating steady state and the internal stability of the closed-loop system.

The performance of the proposed feedforward/feedback control methodology was tested in terms of rejection of step changes at the two measurable disturbances. The process was initially assumed to be at steady state. At time $t=1 \mathrm{~h}$ a step change at the inlet monomer concentration $C_{m_{i n}}$ was applied, from 6 to 5 $\mathrm{kmol} / \mathrm{m}^{3}$. The process was allowed to reach a new steady state, and at time $t=6 \mathrm{~h}$ a step change at the inlet temperature $T_{i n}$ was applied, from 350 to $345 \mathrm{~K}$.

Table 1. Kinetic Parameters

\begin{tabular}{llll}
\hline \multicolumn{1}{c}{$i_{j}$} & $Z_{i_{j}}$ & $E_{i_{j}}$ & Reference \\
\hline$T_{c}$ & $3.8223 \times 10^{10} \mathrm{kmol} / \mathrm{m}^{3} \cdot \mathrm{h}$ & $2.9442 \times 10^{3} \mathrm{~kJ} / \mathrm{kmol}$ & Schmidt and Ray, 1981 \\
$T_{d}$ & $3.1457 \times 10^{11} \mathrm{kmol} / \mathrm{m}^{3} \cdot \mathrm{h}$ & $2.9442 \times 10^{3} \mathrm{~kJ} / \mathrm{kmol}$ & Schmidt and Ray, 1981 \\
$I$ & $3.7920 \times 10^{18} \mathrm{~h}^{-1}$ & $1.2877 \times 10^{5} \mathrm{~kJ} / \mathrm{kmol}$ & Tobolsky and Baysal, 1953 \\
$P$ & $1.7700 \times 10^{9} \mathrm{kmol} / \mathrm{m}^{3} \cdot \mathrm{h}$ & $1.8283 \times 10^{4} \mathrm{~kJ} / \mathrm{kmol}$ & Mahabadi and O'Driscoll, 1977 \\
$f_{m}$ & $1.0067 \times 10^{15} \mathrm{kmol} / \mathrm{m}^{3} \cdot \mathrm{h}$ & $7.4478 \times 10^{4} \mathrm{~kJ} / \mathrm{kmol}$ & Stickler and Meyhoff, 1978 \\
$f^{*}=0.58$ & & & Tobolsky and Baysal, 1953 \\
\hline
\end{tabular}


Table 2. System Parameters and Steady-State Values

\begin{tabular}{rlrll}
\hline$F$ & $=1.00$ & $\mathrm{~m}^{3}$ & $F_{I}=0.01679$ & $\mathrm{~m}^{3} \cdot \mathrm{h}^{-1}$ \\
$V$ & $=0.1$ & $\mathrm{~m}^{3}$ & $R=8.314$ & $\mathrm{~kJ} \cdot \mathrm{kmol}^{-1} \cdot \mathrm{K}^{-1}$ \\
$\rho$ & $=866$ & $\mathrm{~kg} \cdot \mathrm{m}^{-3}$ & $M_{m}=100.12$ & $\mathrm{~kg} \cdot \mathrm{kmol}^{-1}$ \\
$C_{I_{i n}}=6.0$ & $\mathrm{kmol} \cdot \mathrm{m}^{-3}$ & $C_{m_{i n}}=8.0$ & $\mathrm{kmol} \cdot \mathrm{m}^{-3}$ \\
$C_{p}=2.0$ & $\mathrm{~kJ} \cdot \mathrm{kg}^{-1} \cdot \mathrm{K}^{-1}$ & $-\Delta H_{p}=57,800$ & $\mathrm{~kJ} \cdot \mathrm{kmol}^{-1}$ \\
$A$ & $=2.0$ & $\mathrm{~m}^{2}$ & $U=720$ & $\mathrm{~kJ} \cdot \mathrm{h}^{-1} \cdot \mathrm{K}^{-1} \cdot \mathrm{m}^{-2}$ \\
$\rho_{w}=1,000$ & $\mathrm{~kg} \cdot \mathrm{m}^{-3}$ & $C_{w}=4.2$ & $\mathrm{~kJ} \cdot \mathrm{kg}^{-1} \cdot \mathrm{K}^{-1}$ \\
$V_{o}=0.02$ & $\mathrm{~m}^{3}$ & $T_{i n}=350$ & $\mathrm{~K}$ \\
$\boldsymbol{y}_{1}^{s p}=25,000$ & $\mathrm{~kg} \cdot \mathrm{kmol}^{-1}$ & $y_{2}^{s p}=335$ & $\mathrm{~K}$ \\
$F_{c w}=3.26363$ & $\mathrm{~m}^{3} \cdot \mathrm{h}^{-1}$ & $T_{w_{o}}=293.2$ & $\mathrm{~K}$ \\
\hline
\end{tabular}

Figures 4-7 illustrate the profiles of the two controlled outputs and the two manipulated inputs, under the assumption of perfect model and perfect measurements. The figures provide a comparison of the output and input responses under

a) The MIMO FF/FB control structure

b) The MIMO GLC structure

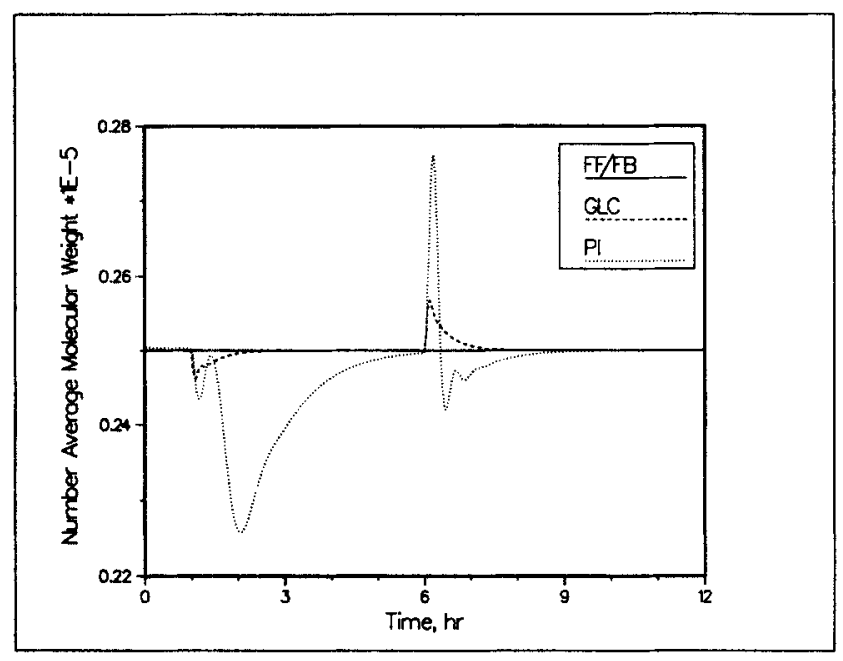

Figure 4. Number-average molecular weight proflles under different control methods.

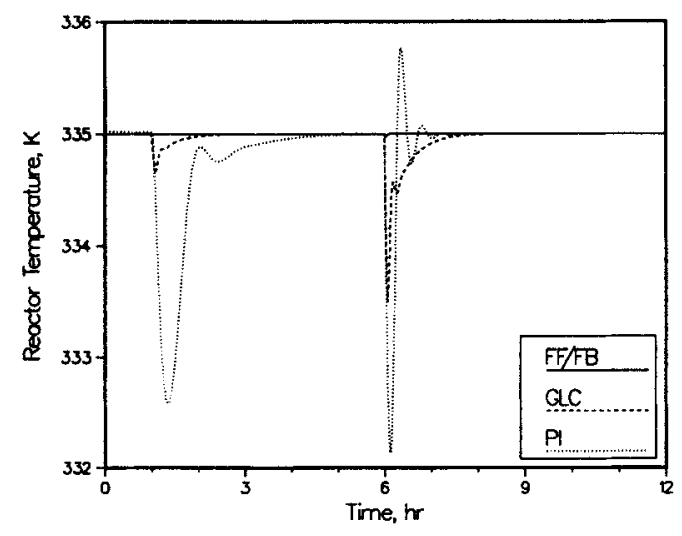

FIgure 5. Reactor temperature proflles under different control methods. c) Two linear SISO PI loops (coolant flow rate/temperature, initiator flow rate/number average molecular weight).

The derivative term in the FF/FB control law (Eq. 17) was approximated by a lead-lag element with transfer function $s /(0.001 s+1)$. In the implementation of the GLC structure, the same values of $\beta_{i k}^{j}$ 's were used as in the FF/FB structure,

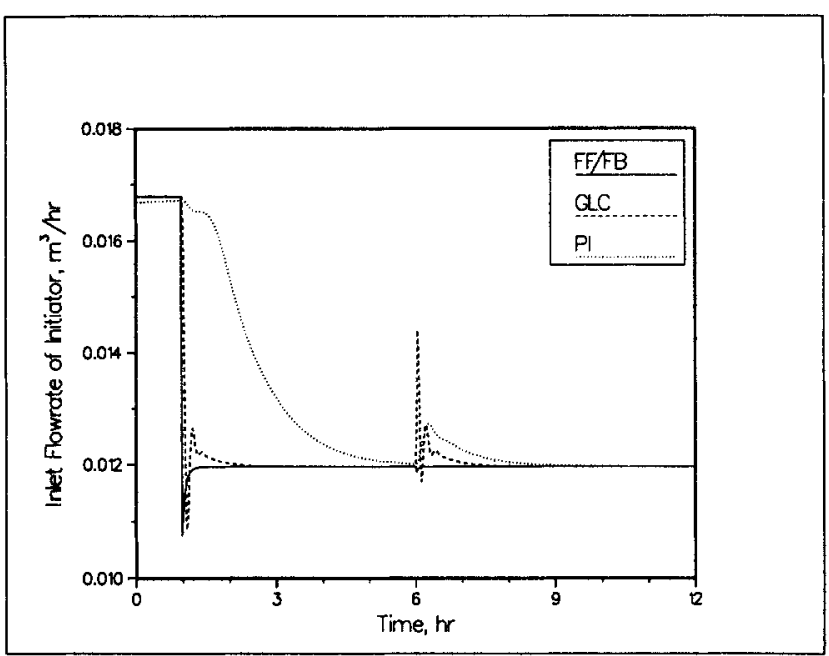

Figure 6. Initiator flow rate profiles corresponding to Figures 4 and 5.

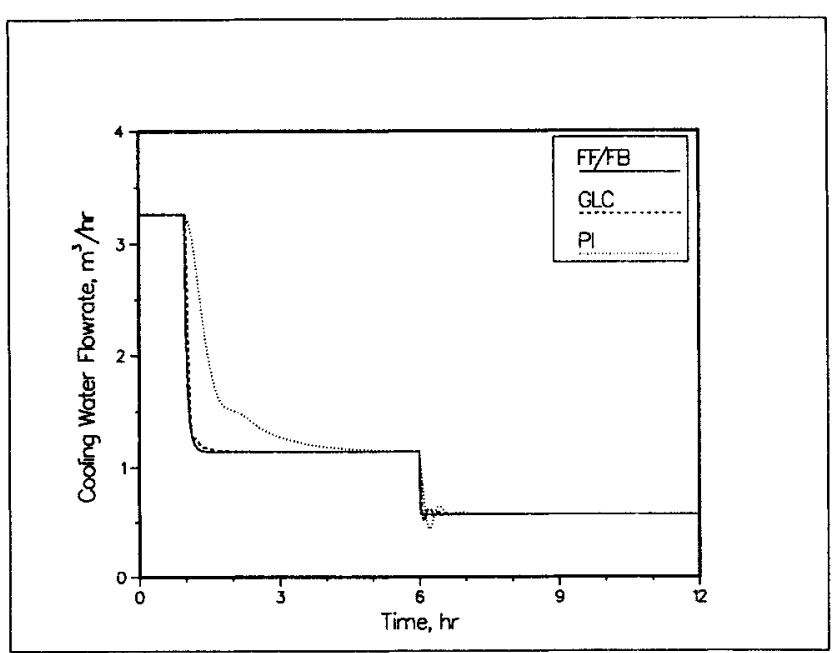

Figure 7. Coolant flow rate profiles corresponding to Figures 4 and 5. 


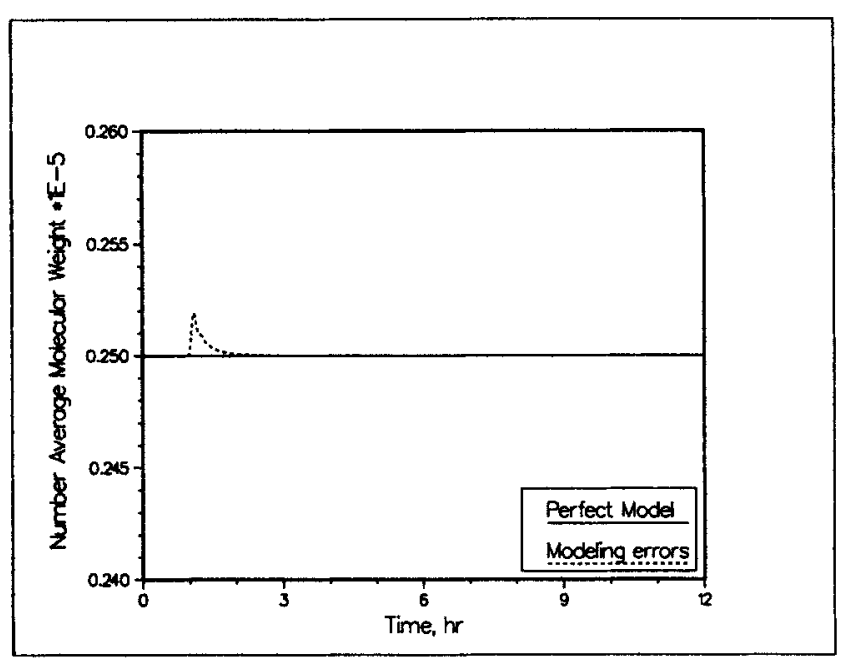

Figure 8. Number-average molecular weight profiles under FF/FB control: effect of model imperfectlons.

while the external linear controllers were chosen as PI controllers with the same settings as in the FF/FB structure. Finally, in the linear control approach, the two SISO PI controllers were tuned through a trial-and-error procedure which resulted in the values $K_{c}=-1 \times 10^{-7} \mathrm{~m}^{3} / \mathrm{h}, \tau_{l}=0.075 \mathrm{~h}$ and $K_{c}=$ $-0.1 \mathrm{~m}^{3} / \mathrm{h} \cdot \mathrm{K}, \tau_{l}=0.075 \mathrm{~h}$, respectively for "best" closed-loop performance. Due to the severe nonlinearity of the process, the response characteristics were found to be very sensitive to the values of the PI controllers' settings.

Clearly, as the theory predicts, the FF/FB control law results in perfect regulation of the outputs, i.e., an obvious improvement of the closed-loop behavior compared with the one under the GLC structure (where no measurements of the disturbances are used in the control law), or the linear PI controllers. In another set of simulation runs, assuming the same disturbance changes as previously, we tested the robustness characteristics of the FF/FB method in the face of modeling error and measurement

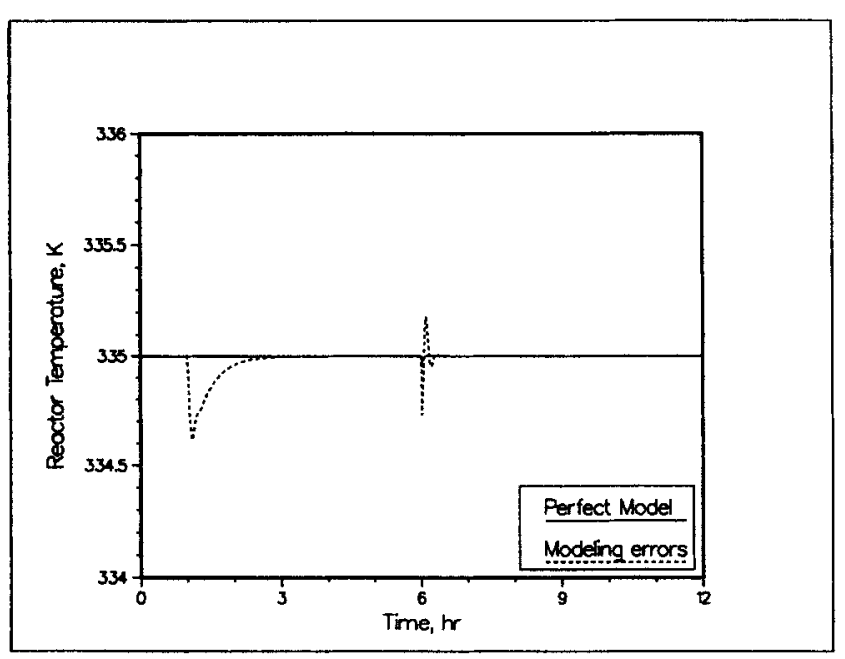

Figure 9. Reactor temperature profiles under FF/FB control: effect of model imperfections.

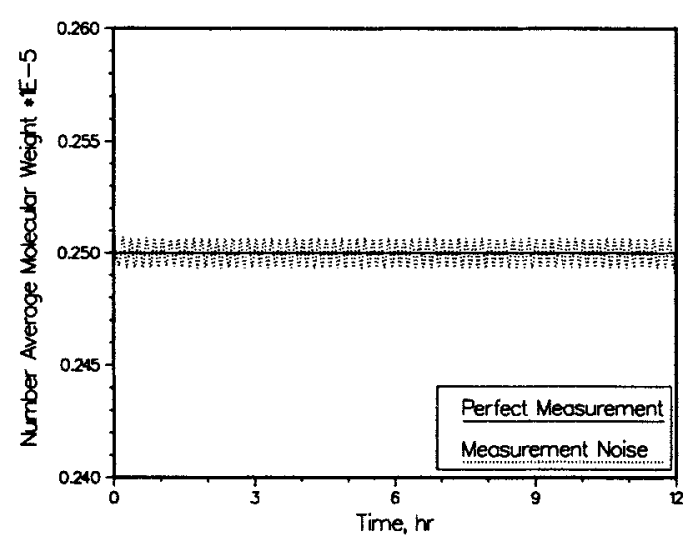

Figure 10. Number-average molecular weight profiles under FF /FB control: effect of disturbance measurement noise.

noise. In particular we compared the closed-loop behavior of the process under

a) Perfect model and perfect disturbance measurements

b) $20 \%$ error in the frequency factor $Z$, and the heat of reaction $\Delta H_{P}$

c) Sinusoidal noise in the measurements of the disturbances $d_{1}$ and $d_{2}$ of amplitudes $0.05 \mathrm{kmol} \cdot \mathrm{m}^{-3}$ and $0.5 \mathrm{~K}$, respectively, and period of oscillation of 10 minutes.

Figures 8 and 9 depict the excellent performance of the FF/FB structure in rejecting the applied step changes in the disturbances for the case when the above model uncertainties exist. As shown in the two figures, even in the presence of the modeling errors, the output profiles are very close to the ones obtained when a perfect model is available. Figures 10 and 11 depict the performance of the FF/FB structure in rejecting the applied step changes when the disturbance measurements are corrupted with the above noise. Clearly, although the output

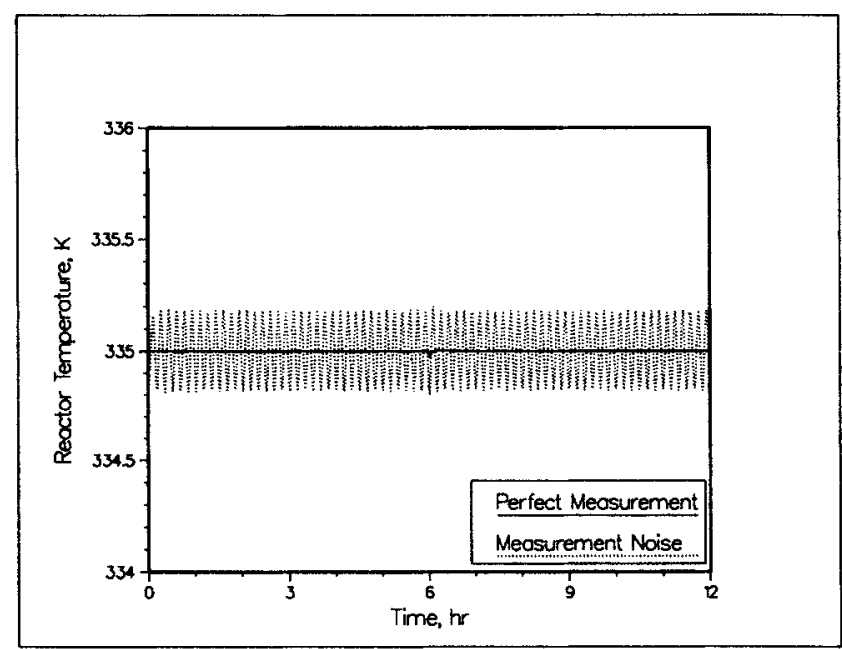

Figure 11. Reactor temperature profiles under FF/FB control: effect of disturbance measurement noise. 
regulation is not perfect in the presence of measurement noise, the proposed method performs very satisfactorily.

\section{Conclusions}

A general synthesis methodology for the feedforward/ feedback control of a large class of multivariable nonlinear processes with measurable disturbances was presented. An appropriate formulation of the concept of relative order, to include disturbance inputs as well as the manipulated input vector, led to a transparent characterization of the regulatory aspect of the problem. A feedforward/state feedback control law that results in complete elimination of the effect of measurable disturbances on the outputs and a linear input/output behavior in the closed-loop system was calculated. The closedloop stability and design objectives were associated with the choice of the adjustable parameters of the control law. The proposed methodology was successfully applied to a continuous polymerization reactor, for the regulation of the number average molecular weight of the product and the reactor temperature, in the presence of measurable disturbances at the composition and temperature of the inlet stream, modeling error and measurement noise.

\section{Acknowledgment}

Financial support from the National Science Foundation through the grant CTS-8912836 is gratefully acknowledged.

\section{Notation}

$$
\begin{aligned}
& A=\text { heat transfer area, } \mathrm{m}^{2} \\
& C_{m}=\text { molar concentration of the monomer, } \mathrm{kmol} / \mathrm{m}^{3} \\
& C_{m_{\text {in }}}=\text { molar concentration of monomer in the mono- } \\
& C_{l}=\text { molar concentration of the initiator, } \mathrm{kmol} / \mathrm{m}^{3} \\
& C_{l_{i n}}=\text { molar concentration of the initiator in the initia- } \\
& \hat{c}_{p}=\text { heat capacity of the reacting mixture, } \mathrm{kJ} / \mathbf{k g} \cdot \mathrm{K} \\
& c_{\mathrm{w}}=\text { heat capacity of water, } \mathrm{kJ} / \mathrm{kg} \cdot \mathrm{K} \\
& D_{0}=\text { molar concentration of the dead polymer chains, }
\end{aligned}
$$

nation by coupling and termination by disproportionation reactions, respectively, $\mathrm{kmol} / \mathrm{m}^{3} \cdot \mathrm{h}$

$Z_{l}=$ frequency factor in Arrhenius equation, for the initiation reaction, $\mathrm{h}^{-1}$

$d=$ vector of disturbance inputs

$f^{*}=$ initiator efficiency

$f, g_{j}, w_{k}=$ vector fields

$h_{i}=$ output scalar field

$r_{i}=$ relative order of the output $y_{i}$ with respect to the manipulated input vector

$s=$ the Laplace domain variable

$t=$ time

$u=$ vector of manipulated inputs

$v=$ external input vector

$x=$ vector of state variables

$y=$ vector of outputs

$y^{s p}=$ vector of set points

$-\Delta H_{P}=$ heat of propagation reaction, $\mathrm{kJ} / \mathrm{kmol}$

$\mathcal{A}_{i}, \mathcal{B}_{i}, \mathcal{E}_{i}=$ partition of disturbances for the output $y_{i}$

\section{Greek letters}

$$
\begin{aligned}
\beta_{i k}^{j}= & \text { parameters of the feedforward } / \mathrm{state} \text { feedback } \\
& \text { law } \\
\tau_{l}= & \text { reset time } \\
\rho= & \text { density of the reacting mixture, } \mathrm{kg} / \mathrm{m}^{3} \\
\rho_{w}= & \text { density of water, } \mathrm{kg} / \mathrm{m}^{3} \\
\rho_{i x}= & \text { relative order of the output } y_{i} \text { with respect to the } \\
& \text { disturbance } d_{k}
\end{aligned}
$$

\section{Math symbols}

$$
\begin{aligned}
\Rightarrow & =\text { implies } \\
\Leftrightarrow= & \text { is equivalent to } \\
E= & \text { belongs to } \\
\varnothing= & \text { intersection } \\
\text { det }= & \text { void set } \\
k e r= & \text { kernerminant of a matrix } \\
d h_{i}= & \text { gradient of a scalar field } h_{i} \\
\mathbb{R}= & \text { real line } \\
\mathbb{R}^{n}= & n \text {-dimensional Euclidean space } \\
L_{f} h_{i}= & \text { Lie derivative of the scalar field } h_{i} \text { with respect } \\
& \text { to the vector field } f \\
L_{f}^{k} h_{i}= & k \text {-th order Lie derivative of the scalar field } h_{i} \\
& \text { with respect to the vector field } f
\end{aligned}
$$

\section{Acronyms}

BIBO = bounded input bounded output

SISO = single input single output

MIMO = multiple input multiple output

GLC = globally linearizing control

FF $/ F B=$ feedforward $/$ feedback

CSTR $=$ continuous stirred tank reactor

\section{Literature Cited}

Congalidis, J. P., J. R. Richards, and W. H. Ray, "Feedforward and Feedback Control of a Copolymerization Reactor," $A I C h E J ., 35$, 891 (1989).

Daoutidis, P., and C. Kravaris, "Synthesis of Feedforward/StateFeedback Controllers for Nonlinear Processes," AIChE J., 35, 1602 (1989a).

Procedforward/State-Feedback Control of Nonlinear Processes," Proc. A.C.C., 1082, Pittsburgh (1989b).

Freund, E., "The Structure of Decoupled Nonlinear Systems," Int. J. of Contr., 21, 443 (1975).

Ha, I. J., and E. G. Gilbert, "A Complete Characterization of Decoupling Control Laws for a General Class of Nonlinear Systems," IEEE Trans. Autom. Contr., AC-31, 823 (1986).

Hirschorn, R. M., "Invertibility of Multivariable Nonlinear Control Systems," IEEE Trans. Autom. Contr., AC-24, 855 (1979).

" "(A, B)-Invariant Distributions and Disturbance Decoupling of Nonlinear Systems," SIAM J. Contr. Optimiz., 19, 1 (1981).

Hunt, L. R., R. Su, and G. Meyer, "Design for Multi-Input Nonlinear 
Systems," Differential Geometric Control Theory, R. W. Brockett, R. S. Millman, and H. J. Sussman, eds., Birkhauser, Boston, 268 (1983).

Isidori, A., A. Krener, C. Gori-Giorgi, and S. Monaco, "Nonlinear Decoupling via Feedback: a Differential Geometric Approach," IEEE Trans. Autom. Contr., AC-26, 331 (1981).

Kravaris, C., and M. Soroush, "Synthesis of Multivariable Nonlinear Controllers by Input/Output Linearization," $A I C h E$ J., 36, 294 (1990).

Mahabadi, H. K., and K. F. O'Driscoll, "Absolute Rate Constants in Free-Radical Polymerization: III. Determination of Propagation and Termination Rate Constants for Styrene and Methylmethacylate," $J$. Macromol. Sci.-Chem., A11, 967 (1977)

Ray, W. H., "Multivariable Process Control-A Survey," Comp. and Chem. Eng., 7, 367 (1983)
- "On the Mathematical Modeling of Polymerization Reactors," J. Macromol. Sci.-Revs. Macromol. Chem., C8, 1 (1972)

Ray, W. H., T. L. Douglas, and E. W. Godsalve, "Molecular Weight Distributions in Copolymer Systems. II. Free Radical Copolymerization," Macromol., 4, 166 (1971)

Schmidt, A. D., and W. H. Ray, "The Dynamic Behavior of Continuous Polymerization Reactors-I," Chem. Eng. Sci., 36, 1401 (1981).

Tobolsky, A. V., and B. Baysal, "A Review of Initiation in Vinyl Polymerization: Styrene and MMA,"J. of Polym. Sci., 11, 471 (1953)

Tsoukas, A., M. Tirrell, and G. Stephanopoulos, "Multiobjective Dynamic Optimization of Semibatch Copolymerization Reactors," Chem. Eng. Sci., 37, 1785 (1982).

Manuscript received Dec. 18, 1989, and revision received July 23, 1990 